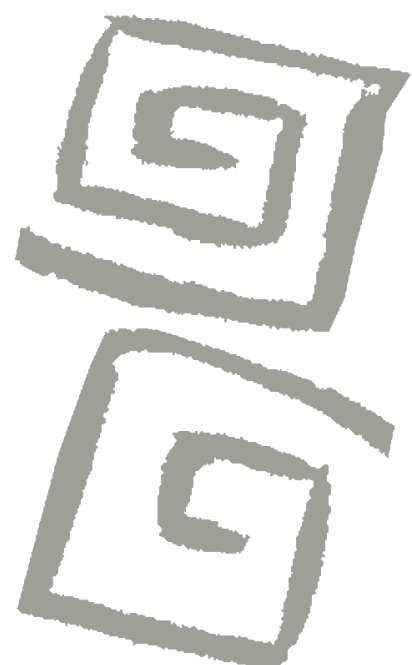

\title{
Entre las religiones y el Estado: el caso de las Expo Promo Salud en la provincia de Buenos Aires
}

\author{
Between religion and the State: the case of the Expo \\ Promo Salud events in the province of Buenos Aires
}

María Pilar García Bossio ${ }^{1}$, Catalina Monjeau Castro ${ }^{2}$

${ }^{1}$ Doctoranda en Ciencias Sociales. Becaria doctoral, Consejo Nacional de Investigaciones Científicas y Técnicas, con sede en el Instituto de Investigaciones en Humanidades y Ciencias Sociales, Universidad Nacional de La Plata, Buenos Aires, Argentina. $\triangle$ iD

${ }^{2}$ Doctoranda en Ciencias Sociales. Becaria doctoral, Consejo Nacional de Investigaciones Científicas y Técnicas, con sede en Centro de Estudios e Investigaciones Laborales, Ciudad Autónoma de Buenos Aires, Argentina. $\square$ (iD)
RESUMEN Las religiones han establecido históricamente formas de regular la vida de sus fieles, que impactan en la concepción del bienestar y la salud. A su vez, espacios estatales han complejizado la definición de salud, incluyendo la no enfermedad del cuerpo, la estabilidad y bienestar social, psicológico, emocional y espiritual. Partiendo de la categoría de cuidado en un sentido amplio, nos proponemos tensionar estas conceptualizaciones a fin de dar cuenta de los puntos de encuentro y discrepancia entre las formas de definición de salud y bienestar por parte de agentes estatales y religiosos. Para ello, desde un enfoque cualitativo, se realizó análisis de contenido de documentos, observación participante y entrevistas formales e informales con agentes estatales y religiosos en el marco de dos eventos organizados, en 2016 y 2017, por la Dirección Provincial de Cultos denominados Expo Promo Salud, espacios instituidos para visibilizar las acciones realizadas por las religiones en el área de la salud en general y del consumo problemático de drogas en particular. A partir de allí esperamos contribuir a las formas de definición discursiva del cuidado, la salud y el bienestar y su posible impacto a la hora de diseñar políticas públicas.

PALABRAS CLAVES Religión; Promoción de la Salud; Bienestar Social; Estado; Argentina.

ABSTRACT Religions have historically established ways to regulate the lives of their followers, impacting conceptions of wellbeing and health. Likewise, state agencies have incorporated a more complex definition of health, including a lack of bodily illness, stability, and social, psychological, emotional and spiritual wellbeing. Using the category of care in a broad sense, we seek to put these conceptualizations into tension in order to identify points of agreement and discrepancy among the ways of defining health and wellbeing by state and religious agents. Using a qualitative approach, content analysis of documents, participant observation and formal and informal interviews with state and religious agents were carried out in the framework of two events held in 2016 and 2017 by the Buenos Aires Provincial Office of Religions under the name Expo Promo Salud. These events were generated to make visible the actions carried out by religions in the area of health in general and problematic drug use in particular. In this way, the article seeks to analyze the ways of discursively defining care, health and wellbeing and their possible impact on the design of public policies.

KEY WORDS Religion; Health Promotion; Social Welfare; State; Argentina. 


\section{INTRODUCCIÓN}

En este artículo, nos interesa preguntarnos sobre la relación entre actores estatales y religiosos en la definición y gestión del bienestar y la salud. Tanto las religiones como los Estados han sabido intervenir en las áreas en cuestión. Las religiones han establecido históricamente formas de regular la vida de sus fieles impactando, de este modo, en la concepción del bienestar ${ }^{(1,2,3)}$ y de la salud ${ }^{(4,5,6)}$. A su vez, organismos internacionales y espacios estatales han complejizado la definición de salud entendiéndola en términos multidimensionales, es decir, incluyendo además de la no enfermedad del cuerpo, la estabilidad y bienestar social, psicológico, emocional y espiritual.

De esta forma, esta concepción amplia de la salud, que parte de la base de pensar a la persona de modo integral, aparece como moneda corriente en el discurso y accionar tanto de instituciones religiosas como de diferentes instituciones sociales ${ }^{(7)}$. En este trabajo, nos proponemos analizar las formas en que el Estado articula, con diversas religiones, determinadas concepciones sobre la salud y el bienestar en términos multidimensionales, impactando en prácticas y gestiones de cuidado que exceden el plano meramente físico y biomédico.

Encontramos relevante el uso de la categoría de cuidado $^{(8)}$ para el análisis de dos eventos organizados por la Dirección Provincial de Cultos de Buenos Aires, nominados como Expo Promo Salud. Estos se esgrimen como espacios de diálogo entre distintas religiones (entre sí y con el Estado), que realizan acciones de cuidado orientadas a la salud, con especial énfasis en el trabajo con usuarios de drogas ilegalizadas ${ }^{(9)}$. Entendemos que el concepto de cuidado nos permite analizar la relación entre actores estatales y religiosos -en la medida en que nos habla de un modo amplio de la gestión de lo común que, en casos como el aquí analizado, persigue como fin la obtención de bienestar- y visibilizar las tensiones, contrapuntos y encuentros en los procesos estudiados.
Por medio de la observación participante en los eventos, del diálogo con los organizadores y asistentes y de la revisión de documentos de instituciones religiosas y estatales referidos a los casos estudiados, esperamos poder indagar en la complejidad de tareas, actividades y discursos que contemplan dimensiones como la "material" y la "espiritual" que hacen a definiciones de la gestión de la salud y el bienestar por parte de agentes estatales y agentes religiosos. Para ello, organizaremos el artículo del siguiente modo. Luego de mencionar los aspectos metodológicos, presentaremos una problematización del concepto de bienestar en vínculo con los estudios sociales sobre el Estado. Posteriormente, nos detendremos en la articulación con lo religioso a partir de la descripción del caso vinculado a la gestión estatal de la Provincia de Buenos Aires. En función de esa descripción general, puntualizamos en tres particularidades de los eventos a analizar, en tanto creemos que el objeto construido nos habilita a pensar el bienestar y la salud desde una mirada que contemple las tensiones y puntos de encuentro entre agentes estatales y agentes religiosos.

\section{ASPECTOS METODOLÓGICOS}

Para realizar este trabajo se recurrió, desde un enfoque cualitativo, a los datos construidos a partir de notas de campo, al análisis de contenido de documentos, observaciones participantes y entrevistas formales e informales con agentes estatales y religiosos en el marco de dos eventos organizados por la Dirección Provincial de Cultos denominados Expo Promo Salud que tuvieron lugar en 2016 y 2017.

Abordamos estos eventos desde una perspectiva etnográfica multilocal(10), en tanto nos permitió seguir las conexiones, asociaciones y relaciones imputables entre los actores a estudiar. Más que realizar un trabajo de campo anclado solo en un espacio delimitado, seguimos los movimientos de un grupo particular de sujetos iniciales, en este caso, 
agentes de la Dirección Provincial de Cultos y las instituciones religiosas que participaron en los eventos en cuestión.

Procurando efectuar un estudio que se base en la comprensión de hechos y significados socialmente construidos, las técnicas de análisis implicaron simultáneamente la recolección de datos, la comparación y revisión constante con las categorías teóricas construidas ${ }^{(11)}$. Como disposición general, la observación participante lideró las situaciones sociales que permitieron construir los datos aquí presentados. La entrevista etnográfica o antropológica ${ }^{(12,13,14)}$, entendida como una relación social que permite acceder a los universos de sentidos de los actores, ordenó el trabajo de campo realizado. Asimismo, siguiendo la propuesta de Gupta ${ }^{(15)}$ para la realización de una etnografía del Estado que pueda dar cuenta de su constitución en términos discursivos por parte de diferentes actores involucrados en este proceso, se complementó la producción de datos realizando un análisis de contenido de documentos de distinto tipo: desde artículos de medios de comunicación producidos por instituciones religiosas y estatales que cubrieron los eventos en cuestión, pasando por folletería y merchandising de las organizaciones religiosas, hasta información recabada a partir de las páginas web de las instituciones participantes.

Las entrevistas semiestructuradas fueron realizadas al funcionario a cargo de la Dirección Provincial de Cultos y al agente con mayor antigüedad en la oficina durante el mes de noviembre de 2016, en las dependencias del organismo. Las entrevistas fueron grabadas tras el consentimiento informado de los actores. Esto se complementó con entrevistas informales en el contexto del trabajo de campo etnográfico, donde se procedió a tomar notas luego de las conversaciones. La dinámica de ambos eventos, con abundante circulación de gente en espacios abiertos, permitió que se realizaran apuntes preliminares en el lugar, para luego extenderlos como notas de campo. Las conversaciones incluyeron al director, a dos agentes de la dependencia (uno encargado de la locución de los eventos y otro de la logística) y a la directora de Cultos del municipio de Florencio Varela. Allí se indagó sobre las impresiones generales del evento (para el caso del segundo se buscó establecer comparaciones), el nivel de convocatoria, la forma de selección de los participantes y su organización, la gestión del tiempo de los eventos, entre otras cuestiones.

Esta estrategia de entrevistas informales se utilizó también con miembros de cultos participantes en las Expo Promo Salud, a partir de la visita a los estands de cada religión. Por medio de estas conversaciones se indagó en las percepciones sobre las actividades realizadas (sobre todo, en términos de salud) y sobre la participación en el evento. El contacto fue con público organizador y asistente que, de manera voluntaria, participó en los estands ofreciendo información sobre sus religiones y las tareas que realizan en torno a la salud. En todos los casos, informamos tanto nuestra identidad como investigadoras como nuestro objetivo de realizar un trabajo académico con los datos producidos en el marco de ese campo. Mantuvimos breves charlas en todos los estands, pasando por algunos de ellos más de una vez. Entendiendo la situación de entrevista como una relación social, cabe remarcar que las conversaciones más fluidas fueron producto de la predisposición de las personas a conversar, en especial, los miembros de la Iglesia de Jesucristo de los Últimos Días, la Iglesia Adventista, la Iglesia Scientology Argentina, y miembros de la Agrupación Social, Cultural y Religiosa Africanista y Umbandista (ASRAU). Estos últimos no tenían un estand propio, pero participaron activamente del evento en San Francisco Solano.

Las nociones sobre salud y bienestar fueron elaboradas a partir de datos construidos en el marco de entrevistas (semiestructuradas y etnográficas) y observaciones, analizados en un proceso dialógico con categorías construidas por las autoras y reescritas en relatos que permitieron identificar regularidades $y$ singularidades en las definiciones compartidas (o no) por nuestros actores. Se obtuvo el consentimiento informado por parte de los actores involucrados en el trabajo de campo. El procesamiento de los datos obtenidos se produjo a partir de la lectura analítica de los 
diarios de campo y entrevistas desgrabadas, sin mediar el uso de software de análisis cualitativo de datos.

\section{ACERCA DE LOS DEBATES SOBRE BIENESTAR Y CUIDADO}

De diferentes maneras, el Estado y las religiones se ocupan de producir, promover y gestionar el bienestar de los sujetos y las poblaciones. Dentro del gran abanico que es el bienestar, la temática de la salud (con sus múltiples dimensiones construidas socialmente) aparece como un área de injerencia importante. Sin embargo, como veremos a continuación, los estudios sobre bienestar han tendido a separar procesos (materiales, físicos, biomédicos, psicológicos, emocionales, espirituales) y actores. La necesaria distinción analítica entre ambos olvida algunas veces el carácter integral que para los actores guardan estos procesos. Por ese motivo, consideramos que la categoría de cuidado desde una perspectiva amplia nos permitirá poner en diálogo estas cuestiones.

En América Latina, si bien el Estado de bienestar no se ha desarrollado como en otros países, sí han existido diferentes instituciones que han desplegado toda una gama de servicios, recursos e infraestructuras proveedoras de bienestar para diferentes sectores de la población. La idea de que la producción de bienestar descansa meramente en prácticas estatales fue desechada por los estudios pioneros de Esping-Andersen ${ }^{(16)}$ cuando incorporó una dimensión más amplia y societal en los procesos de producción de este. En lugar de hablar de Estado de bienestar, el autor propuso el concepto de régimen de bienestar que se refiere, de modo general, a "los modos en que se reparte la producción de bienestar entre el Estado, el mercado y las familias"(16).

Adelantado et al. ${ }^{(17)}$ plantearon la necesidad de incorporar el modelo propuesto por Esping-Andersen a las asociaciones formales y grupos informales comunitarios o al sector voluntario, como cuarto generador de bienestar. Allí podemos incluir las acciones y tareas de las instituciones religiosas que tienden a la producción de bienestar (material y espiritual) en tanto configuran concepciones y prácticas que tienden a la búsqueda y alcance de bienestar, entendido como integral.

A partir de algunos de los debates sobre regímenes de bienestar -por solo mencionar algunos de ellos-, la academia incorporó la noción de gestión de los cuidado, la cual se ha analizado de manera profunda en relación con la distribución social del cuidado entre las familias, el Estado, el mercado ${ }^{(18)}$ y el tercer sector o el ámbito comunitario ${ }^{(19,20,21,22,23)}$. Lejos de entender que las relaciones de cuidado se dan exclusivamente en momentos de dependencia asociados a ciertos estadios vitales y a la reproducción de la vida ${ }^{(24)}$, aquí partimos de una definición amplia de la categoría de cuidado. Esta supone atender a la multiplicidad de relaciones involucradas en el cuidado que involucra, siguiendo a Tronto ${ }^{(25)}$, todas las actividades que hacemos para mantener, continuar y reparar tanto nuestros cuerpos como nuestros "sí mismos" (selves) y las relaciones sociales y ambientales en las que nos hallamos inmersos ${ }^{(25,26,27)}$.

En este punto cabe agregar que, si bien tanto el sentido común como gran parte de la bibliografía especializada ligan unilateralmente el cuidado a específicas relaciones sociales ancladas en la obtención de bienestar para las personas, tener una mirada amplia y relacional sobre el cuidado implica incluso incorporar actividades que pueden provocar, al menos en el corto o mediano plazo, malestar en las personas. En este sentido, Álvarez y Auyero ${ }^{(28)}$ señalan cómo en contextos de violencia interpersonal, para lidiar con riesgos que atentan con la vida y el bienestar personal, se recurre a una "ética popular de cuidado" que -por medio de, por ejemplo, el dolor, la angustia, el miedo y el uso de violencia física- intenta evitar la producción de una situación concebida en términos morales como peor. Este tipo de prácticas asociadas a la producción de un malestar inmediato en las personas, lejos de quedar en el ejercicio de una mera crueldad, es entendido por los autores y por los mismos actores estudiados 
como una expresión de cuidado, como una manera de cuidar a otros y como un medio para, en última instancia, alcanzar el bienestar.

Retomando a Al-Mohammad y Peluso ${ }^{(29)}$, en las superposiciones y relaciones dadas en la vida cotidiana, reside una ética del cuidado del "suelo áspero". Estos autores esbozan una lectura analítica por medio de la cual dan cuenta de cómo en la sociedad iraquí posinvasión 2003 conviven situaciones de dolor, sufrimiento y malestar con pequeñas prácticas mundanas basadas en la alegría, el amor y el cuidado interpersonal que habilitan rutinas que hacen más soportable la vida. Esto nos permite reflexionar acerca de la importancia de desnaturalizar el concepto de cuidado como linealmente ligado a estados de bienestar.

Por ello es que sostenemos la relevancia de mirar los fenómenos estudiados a la luz de una categoría que dé cuenta de cómo las personas viven y experimentan (en complejas articulaciones institucionales y que involucran variedad de prácticas y actividades sociales) relaciones de cuidado. Si entendemos el bienestar como un estado definido socialmente que involucra, de acuerdo a los diferentes actores, dimensiones tales como la física, la psicológica, la emocional y la espiritual, los cuidados condensarían las diferentes actividades y prácticas que habilitan un acercamiento a dicho estado. De este modo, la categoría de cuidado resulta pertinente en tanto permite captar la variedad de prácticas ligadas a, por ejemplo, la solidaridad, la disciplina, la violencia, los rezos y las oraciones, los tratamientos de salud, las prácticas de sanación, las actividades de prevención y tratamiento de consumo problemático de drogas, la ayuda social, el asistencialismo, entre otros.

A partir de esta categoría amplia de cuidado y de su relación con la forma de concebir la salud y el bienestar, nos proponemos analizar el caso de una serie de eventos organizados por una dependencia de la provincia de Buenos Aires que contaron con la participación de diversas instituciones religiosas. Desde allí, abordaremos las tensiones y puntos de encuentro existentes en la forma de conceptualizar la salud por parte de agentes estatales y religiosos y su vínculo con las maneras en que estos actores proponen gestionar el cuidado.

\section{Las Expo Promo Salud}

En este apartado, describimos el caso a analizar para posteriormente profundizar en algunas características particulares de los eventos en cuestión, que hacen a la relación entre bienestar, salud y cuidado, enfocándonos en, por un lado, la participación de dos instituciones religiosas no católicas y, por otro, en el accionar de grupos religiosos en torno al consumo problemático de drogas.

Las Expo Promo Salud fueron organizadas por la Dirección Provincial de Cultos de la provincia de Buenos Aires. Esta es una dirección del poder ejecutivo provincial, dependiente de la Secretaría General de la Gobernación. Tiene entre sus funciones principales establecer vínculos con las diversas religiones en el territorio provincial, promoviendo actividades concebidas como de impacto social positivo. Entre las tareas que desempeña, esta oficina realizó dos eventos (uno en noviembre de 2016 en Florencio Varela y otro en mayo de 2017 en San Francisco Solano, en el sur del Gran Buenos Aires) como parte de sus acciones de cooperación y diálogo interreligioso.

El objetivo propuesto $-y$ replicado por los medios de comunicación- fue "presentar distintas alternativas vinculadas con la prevención de las adicciones y la salud desde su aspecto físico, mental, emocional y espiritual, promoviendo los hábitos saludables"(30). De esta forma, la Dirección buscó cumplir con una de sus misiones organizacionales, según la cual se intenta continuar, sostener y profundizar el diálogo con referentes de todas las religiones, en tanto estas "ejercen una transformación positiva sobre la realidad social"(31).

Podemos ver ya aquí la intención del Estado de generar un espacio de accionar conjunto y articulado con instituciones religiosas de la sociedad civil de manera de intervenir 
en la gestión de lo común como forma de acceso al bienestar. Asimismo, es dable destacar la elección del área de salud (y del consumo problemático de drogas en particular, como veremos más adelante) como espacio desde del cual llevar adelante esta articulación. Volveremos y profundizaremos sobre ello en los próximos apartados.

En los eventos estudiados, participaron distintas denominaciones religiosas entre las que se encontraban la Iglesia católica, a través de la diócesis de Quilmes, la Iglesia Adventista del Séptimo Día, la Iglesia de Jesucristo de los Últimos Días, la Iglesia Evangélica Bautista, la Santa Iglesia del Señor, la Asociación de la Iglesia de Dios, la Iglesia Scientology Argentina, y diversos consejos pastorales evangélicos (Federación de Iglesias Cristianas Evangélicas, Consejo Pastoral del Partido de Quilmes, Consejo Unido de Pastores de Solano, Pastores Unidos para Ayudar Ministerio Interdenominacional, Espacio Interreligioso de Diálogo y Acción en Materia de Promoción Social).

A su vez, se contó con la presencia de diferentes agentes estatales: autoridades municipales (en ambos eventos, se hicieron presentes los directores de cultos de Quilmes y Florencio Varela) y otros organismos provinciales, como la Subsecretaría de Determinantes Sociales de la Salud y la Enfermedad Física, Mental y de las Adicciones; las Direcciones Provinciales de Deporte y Juventud; el Organismo para la Niñez y Adolescencia y la Subsecretaría de Adicciones. En la segunda edición, se incorporaron el Organismo Provincial para el Desarrollo Sostenible (OPDS) y el Registro Provincial de Organizaciones de la Comunidad. En esta última edición, se hizo presente el intendente de la localidad de Quilmes.

La participación de las instituciones religiosas fue, en ambos casos, de inscripción voluntaria, si bien la Dirección realizó algunas invitaciones puntuales -conforme a las relaciones que ya mantiene con algunos cultos- fundamentalmente a la Iglesia Católica y a algunas denominaciones protestantes, como la Iglesia Adventista. La presencia de la Iglesia católica fue importante, en especial en la primera edición, en la que al finalizar se utilizó el escenario para celebrar la XXI Misa de la Esperanza, como parte del aniversario de la diócesis de Quilmes (que incluye a las localidades de Quilmes, Berazategui y Florencio Varela).

En este punto, nos parece importante recordar que, en Argentina, la Iglesia Católica posee un estatuto legal diferencial frente a otras iglesias, dado por su sostenimiento constitucional y por su carácter de persona jurídica pública ${ }^{(32)}$. Si bien esto no impactó de forma directa en el modo en que se llevaron adelante ambos eventos, sí nos habla de la pervivencia de una posición jerarquizada de esta Iglesia sobre otras, incluso cuando pudimos apreciar una creciente participación evangélica en estos espacios. Además, la centralidad dada a la Iglesia católica en el uso del escenario de esa primera edición generó malestar en la comunidad adventista, lo que hizo que esta congregación le informara a la Dirección su decisión de no participar en la siguiente.

De todos modos, la convocatoria abierta a los eventos tuvo el objetivo, según lo relatado por los propios agentes de la Dirección, de volver los eventos lo más inclusivos posibles, brindando la posibilidad de acercarse a comunidades que no tuvieran un vínculo preexistente con el Estado. Desde lo observado en el campo, esto propició una ligera apertura a otras religiones, contando, en la segunda edición, con la participación de la Iglesia Scientology Argentina y miembros de la Agrupación Social, Cultural y Religiosa Africanista y Umbandista (ASRAU), que posee una escuela en San Francisco Solano.

Aquí podemos observar una primera tensión entre el Estado provincial y las religiones que se relacionan con él, al querer fomentar la cooperación religiosa en la promoción del bienestar social general, incorporando incluso religiones socialmente estigmatizadas $^{(33)}$, como el umbandismo, pero sin tocar la situación de privilegio de la Iglesia católica. Esto lleva a la Dirección a posicionarse como articuladora de relaciones dispares, que si bien no generan tensión en el momento de la exhibición, sí producen reclamos posteriores. 
La dinámica de ambos eventos fue semejante entre sí, con carpas en espacios públicos al aire libre, la primera edición contó con un espacio abierto más grande que posibilitó, como veremos, la puesta en práctica de otras dinámicas. Cada grupo religioso tenía uno o varios estands; además, había un par de puestos de comida, uno de la Cruz Roja, un laboratorio del Ministerio de Salud de la provincia de Buenos Aires y un escenario, en el que tocaron de manera continua bandas de rock cristiano y grupos de música popular católicos. Desde ese escenario, el director de Cultos agradeció la asistencia de forma general, mientras un locutor anunció detalladamente a los participantes, a la vez que animó y presentó las bandas. En cuanto a los estands, cada grupo religioso tenía asignado uno o más gazebos, en los que había dos o tres personas integrantes de las organizaciones, casi siempre con remeras distintivas, que explicaban el trabajo y actividades realizados en temáticas de salud y atención a la drogodependencia. Allí, brindaban folletería e incluso, en algunos casos -mayormente en organizaciones católicas-, ofrecían algún producto realizado por las personas en recuperación de situaciones de drogodependencia.

Las personas circulaban entre los estands y permanecían algunos minutos en cada uno de ellos. No notamos tensiones manifiestas entre los distintos grupos en la convivencia, así como tampoco una gran circulación de un estand a otro por parte de los miembros de las diversas religiones. En ambos casos, circuló gente de la localidad donde se realizó el evento, que recibía folletería y escuchaba a las bandas que animaban, sin que hubiera más de una decena de personas a la vez apreciando los shows, las cuales no permanecían mucho tiempo.

Esto pareciera indicar que las Expo estudiadas se constituyen como espacios de encuentro, en los que, si bien no se producen situaciones de discriminación o tensión en el momento, no llega a darse un clima de intercambio interreligioso fluido. Así, el nexo entre los actores religiosos parece ser, fundamentalmente, la acción estatal, que produce la situación y la temática de la salud como motivo para el encuentro al reconocer en ellos una "acción positiva" frente a la problemática del consumo de drogas.

Cabe destacar, en este punto, que la Dirección, según lo expresado por su director en el marco de una entrevista personal, busca establecer un espacio que trascienda el diálogo religioso para constituir una cooperación activa de las religiones entre sí y con el Estado en el territorio y que, si bien es la Dirección quien decide llamar a instituciones religiosas para que presenten al público sus actividades, intervenciones y discursos sobre la salud, la confluencia de estos actores específicos en torno a la temática nos habla de una intervención que ubica, a la vez, a los agentes religiosos y a los estatales como productores legítimos del cuidado de la salud. $\mathrm{Y}$ aunque los grados de legitimidad no sean los mismos (como veremos en algunas de las tensiones que analizaremos a continuación), no deja de ser importante marcar esta producción discursiva.

Habiendo descrito las características generales de ambos eventos y los principales actores involucrados, nos proponemos profundizar a continuación en las particularidades que hallamos interesantes de cada una de las ediciones en cuestión, en tanto nos permiten reflexionar sobre las tensiones y puntos de encuentro entre agentes estatales y religiosos a la hora de definir el bienestar y la salud. Para ello, analizaremos (en los dos siguientes apartados) el accionar de dos religiones no católicas, para luego (en el último) focalizar en la participación de religiones cristianas en la prevención de adicciones. Tomamos la decisión expresa de escoger el accionar de la Iglesia adventista, en la primera edición, y de la Iglesia Scientology, en la segunda, fundamentalmente por dos motivos.

En primer lugar, las situaciones dadas en el mismo trabajo de campo hicieron emerger las intervenciones de ambas iglesias como interesantes a los ojos de las autoras ya que, en un doble registro, a sus miembros les importaba visibilizar su accionar, mostrándose muy predispuestos al diálogo y, a su vez, condensaban particularidades interesantes de retomar de cara al objetivo del presente artículo. 
En segundo lugar, ambas iglesias pueden ser consideradas como minoritarias, en términos cuantitativos, y muchas veces estigmatizadas, frente a la posición de privilegio de la Iglesia católica. Esta estigmatización produce discursos sociales que asocian a estas religiones con la producción de malestares personales y sociales, en la medida en que son consideradas como "sectas" que producen "lavados de cerebro" sobre sus fieles ${ }^{(33)}$. De esta manera, poner foco en las intervenciones, accionares y producciones discursivas que estas instituciones religiosas elaboran, nos permite preguntarnos por sus concepciones sobre el bienestar y la salud y por cómo estas se relacionan con las construidas desde el Estado en el marco de las Expo Promo Salud estudiadas.

Finalmente, presentaremos el caso de diferentes grupos cristianos que, en ambas ediciones de las Expo, expusieron su trabajo sobre la prevención, atención y tratamiento de la drogodependencia, para tematizar allí las tensiones y puentes que se establecen con el Estado en las definiciones acerca de la gestión de la salud y el bienestar. Cabe destacar que no será de nuestro interés estudiar en sí diferentes tipos de comunidades terapéuticas sino, más bien, poner el foco -en el mismo ejercicio analítico que el propuesto en los primeros dos casos- en las definiciones de salud y bienestar, presentando los dispositivos institucionales religiosos de las Expo para el tratamiento, la prevención y el cuidado en situaciones de consumo problemático de drogas.

\section{Primera edición: participación adventista y acercamiento a Dios como forma de bienestar}

$\mathrm{Si}$ analizamos ahora las particularidades de cada uno de los eventos, podemos destacar en la primera edición una actividad de la Iglesia adventista, a cargo de su área de intervención en materia de salud (denominada por esta como "Ministerio de salud adventista"). Bajo el nombre de "Expo vida + sana", la iglesia en cuestión organizó un recorrido de ocho pasos delimitados por una serie de gazebos, sumado a una actividad de gimnasia aeróbica desarrollada frente a estas carpas. Esta actividad dentro del evento organizado por la Dirección de Cultos forma parte de distintas propuestas en torno a la salud que la Iglesia adventista realiza de forma regular, no siempre en vínculo con espacios estatales. La periodicidad de este tipo de propuesta se hace evidente desde el momento en que notamos que dicha iglesia posee en su página web un manual para realizar este tipo de tareas, con el nombre de "Feria de Salud"(34).

Es interesante destacar que, en ese manual la iglesia recomienda acudir a apoyo estatal para la realización de las actividades, no solo en términos económicos, sino también mediante el acceso a profesionales biomédicos de la salud, material médico descartable, etcétera. Se propone que el vínculo se establece con el municipio o con secretarías como la de Salud y Educación, mostrando cómo en el nivel de la política provincial y municipal las relaciones entre líderes religiosos y políticos son más cercanas que en el nivel nacional, lo que provoca una mayor influencia de las autoridades religiosas sobre los poderes ejecutivos locales ${ }^{(35)}$. Esto se evidenció con claridad en nuestro caso, dado que esta Feria fue una de las actividades con más personas participantes dentro de la Expo, tanto por parte de visitantes ocasionales como de miembros de otras religiones.

Como hemos mencionado, el recorrido constaba de ocho "paradas", en las que se daban charlas sobre la salud en general, se tomaba la presión, la glucosa y la capacidad pulmonar, se realizaban ejercicios físicos y masajes y se finalizaba con un test de "edad biológica" en el que se comparaba la edad real con el desgaste vital medido en las estaciones previas. En ese momento, el voluntario adventista introducía una pequeña charla sobre la vida espiritual, entregando un libro de la organización, titulado "Viva con esperanza. Secretos para tener salud y calidad de vida".

Podemos ver aquí con claridad cómo un primer acercamiento desde una concepción al parecer unilateralmente biomédica de la salud, va orientándose progresivamente a un 
abordaje entendido como integral: a la vez que lo espiritual pasa a ocupar un lugar central para acceder al bienestar, lo material es reconceptualizado a la luz de esta misma lógica.

Si pensamos en este juego en torno al bienestar como salud física y como salud espiritual, no debemos olvidar otro elemento importante: para los adventistas estos espacios pueden llevar, en última instancia, a un encuentro con Dios. Como indica el manual de la Feria, esta es vista como

...una oportunidad de entrar en contacto con muchas personas con problemas. Muchos recibirán beneficios inmediatos al poner en práctica lo que han aprendido, pero para otros la Feria puede ser el comienzo de un vínculo de amistad y confianza, que puede Ilevarlos a asistir a otros seminarios relacionados con la salud, la familia, lo que a su vez puede guiar a encontrar a Dios. ${ }^{(36)}$

De esta manera, en la medida en que resulta imposible disociar en esta cosmovisión una dimensión "propiamente religiosa" de otra "propiamente material", este momento de transmisión de la fe puede leerse como una forma de gestionar el cuidado desde una mirada integral, que abarca la presencia de elementos en tres dimensiones: una material (del cuidado corporal), una de relaciones sociales (redes de soporte y apoyo mutuo) y una espiritual (de contacto con lo numinoso y de acceso a un bienestar concebido en esos términos). Podemos apreciar la existencia de estas formas similares de gestión del cuidado en algunas prácticas de la Iglesia católica en las que, como señala Giménez Béliveau, los procesos de atención y contención relacionados con la salud tienen también importancia como forma de (re)establecer vínculos con la fe y de allí con la comunidad ${ }^{(1)}$.

No debemos olvidar aquí que, en el caso que estamos estudiando, la presencia del Estado, es la que garantiza, organiza y promueve el evento en cuestión. Si tenemos esto en consideración, podemos dar cuenta del rol del Estado en este contexto como habilitador en última instancia de espacios para la promoción, no solo de la salud o del bienestar, sino también de determinadas creencias, prácticas y definiciones religiosas.

\section{Segunda edición: Iglesia Scientology y la tensión de las definiciones de salud y bienestar}

De la segunda edición de la Expo Promo Salud, nos interesa destacar la presencia de otras religiones con menor incidencia en el espacio público local, cuyas concepciones de salud y bienestar tensionan con las existentes en el Estado. Para ello analizaremos la presencia de la Iglesia Scientology Argentina a partir de la segunda edición de la Expo Promo Salud. Este caso nos resulta interesante, además, porque es una religión que se acerca al Estado, es decir, que se cuenta entre los cultos que no han sido invitados previamente, sino que participa a partir de la convocatoria de la Dirección Provincial.

Esta Iglesia, originada en EEUU, se define a sí misma como una religión que sostiene la preeminencia del espíritu como el elemento esencial de la condición humana, siendo el hombre más que la mera suma de su cuerpo y su mente. Para esta cosmovisión, al llegar a la comprensión de la verdadera naturaleza espiritual personal se produce la relación con el entorno, lo material y el "Ser Supremo"(37). Cabe destacar que gran parte de la difusión de información de esta iglesia se encuentra en habla inglesa, con videos subtitulados e información traducida a un español sin marcas ni referencias locales.

Como ya hemos mencionado, la Iglesia Scientology contó durante el evento con un espacio amplio, si bien no fue promocionada su participación en los flyers o la gacetilla de prensa circulada por la Dirección de Cultos unos días antes de la Expo. En este lugar, la iglesia desplegó en dos carpas un estand con materiales sobre los principios fundamentales de su cosmovisión y una amplia gama de volantes y videos de difusión en torno a la peligrosidad del consumo de drogas.

Un elemento a destacar es que esta iglesia no solo se opone al consumo de las drogas 
ilegalizadas en el país, sino también a otra serie de fármacos, como analgésicos, antidepresivos y estimulantes, que son reconocidos por el Estado como drogas legales. En efecto, la folletería repartida en el evento, producida por una fundación estadounidense y de carácter genérico para distintos contextos de habla hispana, advierte sobre los peligros del consumo de distintas drogas, varias de escasa o nula presencia en el contexto del conurbano bonaerense, poniendo en pie de igualdad drogas legalizadas e ilegalizadas. Esto lleva a un proyecto preventivo amplio, en el que el foco está puesto en evitar el inicio del consumo a través de la educación. Se diferencia así de otras iglesias que presentan en sus estands sus terapias de recuperación. Para esta religión la solución a una vida signada por las drogas es la misma que para otros problemas vitales: adoptar los principios de Scientology.

En vínculo con lo dicho y sobre la base de la información recabada en el campo, la lógica de sanación subyacente en la intervención de la iglesia en cuestión, consistiría en un acercamiento individual de la persona a esta religión, mediante el cual, solo a través de la adopción de los principios scientology, se llegaría al conocimiento del espíritu, que permitiría el fin del consumo y el alcance de una buena salud y, por tanto, del bienestar.

Si miramos esta dinámica a partir de la categoría de cuidado, notamos un foco en el papel individual en una lógica de autocuidado, con una preeminencia de la dimensión espiritual. La construcción discursiva, en este punto, implica una promoción del cuidado de sí a partir de la introyección de principios y valores propios de la institución Scientology. Esta lógica jerarquiza la dimensión espiritual sobre la material y la de las relaciones sociales.

Nos parece importante, en este punto, aclarar que aquí no estamos centradas en presentar o analizar lo que las personas realmente hacen. Estos datos, enfocados en una dimensión representacional (más que práctica o pragmática) y construidos a partir de análisis de discursos (tanto de lo escrito en los materiales de difusión, como, sobre todo, de lo dicho en el marco de las entrevistas en el campo), dan cuenta justamente de las elaboraciones discursivas que conciben al individuo como el agente clave en la lógica de la gestión del cuidado, la salud y la sanación.

Esto no supondría, a priori, una contradicción con la forma en que el Estado está concibiendo el bienestar, en la medida en que también lo hace a partir de una concepción integral de la salud. Sin embargo, sí existe una tensión si nos concentramos en la clasificación que cada una de estas instituciones elabora sobre el consumo problemático de drogas. El hecho de que la Iglesia Scientology vea a los fármacos recetados como drogas que debieran estar ilegalizadas, nos hace preguntarnos por el carácter fragmentario del aparato estatal. Mientras, por un lado, el Estado legaliza este tipo de medicaciones con protocolos específicos, por otro, en pos de asegurar la diversidad religiosa, habilita espacios en eventos propiciados por él a una religión que cuestiona estas prácticas y principios legitimados. Dar cuenta de esto nos ayuda deconstruir la idea de que existe un Estado que elabora homogéneamente definiciones seculares y secularizadas de la salud.

\section{Estado y religiones en torno a la drogodependencia: clasificaciones de cuidado e intervención terapéutica}

Ambas ediciones de la Expo Promo Salud fueron estructuradas en torno a la temática de la salud en general, pero con un fuerte énfasis en las comunidades religiosas que trabajan sobre consumos problemáticos de drogas. En este apartado, nos concentraremos en señalar algunas de las particularidades de los grupos religiosos que formaron parte de ambos eventos y que tienen como tarea principal la prevención, atención y recuperación de situaciones de drogodependencia, entendiéndolas como formas de gestión y prácticas de cuidado. Tal como se mencionó anteriormente, no es nuestro propósito analizar en sí los dispositivos de tratamiento al consumo problemático de drogas, sino más bien reconstruir los discursos en torno a la salud y al bienestar de las propuestas terapéuticas de las comunidades en el marco de las Expo. 
Como ya ha sido indicado por la bibliografía que trabaja sobre comunidades terapéuticas, situaciones de drogodependencia y grupos religiosos ${ }^{(38,39,40,41,42,43)}$, las religiones ocupan un espacio en esta área de intervenciones públicas sobre la salud. Parte de su legitimidad se debe a una particular expertise a la hora del tratamiento y a una inserción sostenida en el tiempo en el trabajo con sectores populares residentes en barrios marginalizados ${ }^{(44)}$, quienes son considerados los principales afectados por este tipo de problemáticas ${ }^{(45)}$.

Esta expertise, sin embargo, no está dada por saberes médicos o por un lenguaje de tipo científico-técnico, como en el caso de la bioética católica ${ }^{(4)}$, sino más bien por desarroIlar un tipo de tratamiento que se supone más efectivo y menos científico, con un anclaje más fuerte en lo espiritual como herramienta de sanación corporal y de alejamiento de las adicciones ${ }^{(45)}$. Esto puede verse con claridad en los momentos de las Expo en que suben al escenario exadictos con trayectorias "exitosas" de recuperación, donde la presencia de Dios en los discursos es una constante, así como también la presentación de estas trayectorias por parte de sacerdotes o pastores.

Esto no le quita al Estado el monopolio de la responsabilidad política sobre la definición de salud y el bienestar y sobre el accionar ante las drogas ilegalizadas, pero sí supone el reconocimiento, por parte de este, de cierta posición de saber de las religiones frente a instituciones sociales seculares en la resolución de este tipo de problemáticas. En este sentido, podemos retomar la postura que para Jones y Cunial(45) tienen los decisores políticos frente a organizaciones de la sociedad civil religiosas:

...como poseedoras de capacidades diferenciales para el abordaje de los consumos de drogas [...] consideradas dueñas de acervos de información mayores a los del Estado con respecto a la población específica a ser atendida por sus dispositivos y con una inserción sostenida en las comunidades afectadas, una gravitación de su acción social en la trama asociativa barrial que Carbonelli [...] conceptualizó como anclaje territorial. Estos rasgos serían evaluados beneficiosos a la hora de ejecutar sus actividades en este campo.

De esta manera, la decisión de la Dirección Provincial de Cultos de visibilizar el trabajo de estos grupos religiosos responde a una situación social preexistente, vinculándose a su vez con la forma de concebir la propia gestión estatal en términos "ejecutivos", en palabras del director. Esto supone la realización de actividades concretas con las religiones -especialmente con la Iglesia católica y la diversidad de denominaciones de las iglesias evangélicas-, facilitando espacios de encuentro con la intención de proyectar acciones conjuntas que trasciendan el diálogo interreligioso. De allí lo novedoso de este tipo de experiencias, en las que el Estado, a través de la Dirección de Cultos, visibiliza a estas comunidades terapéuticas. Si bien el objetivo central explícito de los organizadores es promover junto al diálogo interreligioso acciones concretas en materia de intervención social, la elección de la temática de la salud, como eje rector de los encuentros, nos habla, como sostenemos, de una forma de concebir la salud y el bienestar de modo, a la vez compartido y tensionado por las religiones y los actores estatales.

Si nos preguntamos qué tipo de comunidades participaron de estos eventos, podemos tomar como referencia la tipología que Camarotti, Güelman y Azparren ${ }^{(9)}$ proponen para programas terapéuticos en instituciones del Área Metropolitana de Buenos Aires (AMBA) que incluyen algún tipo de abordaje espiritual o religioso. A partir de allí, podremos cruzar las dimensiones del cuidado en términos ampliados que planteamos en este trabajo, estableciendo tres grandes grupos participantes de ambas ediciones de las Expo. $\mathrm{Si}$ bien no es nuestro objetivo ahondar en las características de las distintas comunidades terapéuticas, esta clasificación nos permitirá profundizar el análisis, en la medida en que nos habilita a presentar de forma más ordenada la multiplicidad de organizaciones y 
maneras de abordar la problemática del consumo de drogas ilegalizadas.

La tipología presentada por los autores citados encuentra dos grandes orígenes del consumo: uno de índole social y otro de índole individual. Desde allí presentan tres formas de concebir al consumidor, lo que lleva a proponer tres modalidades de tratamiento centradas en la recuperación del sentido de vida, en la inclusión social o en el acompañamiento ante la enfermedad crónica de la adicción (mientras las dos primeras responderían a causalidades de origen social, la tercera, estaría centrada en la individual). Esto, a su vez, impacta en las formas de financiamiento, lo que puede constituirse en un indicador del reconocimiento estatal ${ }^{(45)}$.

En cualquiera de estas tres modalidades, encontramos una específica propuesta de gestión del cuidado hacia los usuarios de drogas ilegalizadas por parte de las religiones, en la medida en que las religiones proponen desplegar, en este espacio de difusión, una serie de estrategias de cuidado no solo materiales, sino también espirituales y sociales ancladas en un diagnóstico del origen de la adicción como espiritual y social.

Podemos dar cuenta de la presencia de estos tres tipos de instituciones en las dos ediciones de la Expo, con una cierta preeminencia de las entidades que ponen el eje en explicaciones de índole social y relacional, que son las que hacen mayor hincapié en el factor espiritual y religioso de la rehabilitación.

Dentro de las instituciones que ven el consumo de drogas como resultado de la pérdida de sentido de la vida en el contexto de la sociedad moderna $y$, por ende, como un problema de índole fundamentalmente espiritual, encontramos en las Expo a los centros evangélicos Reto a la Vida y Remar, pudiendo incluir aquí también a algunos grupos católicos. Este tipo de organizaciones son las que hacen más eje en la impronta religiosa, y remarcan la importancia de un cuidado que, desde lo espiritual, articule con lo material y lo social, a partir de la creación de "comunidades de vida", con tratamientos prolongados y abstención total del consumo.
El tratamiento es gratuito y se autofinancia, pues al no trabajar con profesionales de la salud no suelen cumplir con los requisitos para obtener apoyo estatal. Para sustentarse, recurren a fondos y donaciones de la Iglesia de pertenencia, así como también a la venta de artículos tales como productos alimenticios (esta acción, de hecho, se realizó en las ediciones de ambas Expo).

A pesar de ser el tipo de instituciones con menos legitimación estatal en términos de financiamiento, en ambos eventos tuvieron una presencia fuerte, con una relación fluida con la Dirección Provincial de Cultos (según pudimos constatar en el seguimiento del accionar de esta dependencia más allá de los eventos estudiados). Consideramos que aquí la Dirección opera desde su función específica de diálogo con las religiones, optando por no reclamar a estas comunidades una adhesión más firme a los principios biomédicos de tratamiento que impulsa el Estado desde sus dependencias específicas de trabajo con adictos.

Un segundo tipo de instituciones presentes son aquellas con fuerte impronta de trabajo por parte de los llamados "curas villeros", sacerdotes católicos que trabajan en lugares marginados de la sociedad, con especial atención a niños y jóvenes en situación de calle. Estos curas ven en lo social la causa de la adicción del consumidor, considerándolo como víctima de condiciones sociales excluyentes. De esta forma, el eje del cuidado está puesto en la construcción de relaciones sociales que mejoren el contexto del adicto, combinando actividades terapéuticas -que incluyen profesionales de la salud- con actividades religiosas. Cabe destacar que estas organizaciones suelen buscar ellas mismas a los adictos para que inicien su internación, lo cual puede entenderse como una práctica de cuidado principalmente anclada en lo material y lo social que, posteriormente, profundiza en lo espiritual. En general, cuentan con algún tipo de financiamiento estatal en forma de becas y subsidios, además de fondos propios de la Iglesia católica ${ }^{(9)}$.

Este tipo de organizaciones tiene una presencia muy fuerte en ambas ediciones de 
la Expo, sobre todo porque son muy activas en la diócesis de Quilmes que, como mencionamos, coincide con la jurisdicción de los municipios propiciantes de los eventos. Autopresentadas como comunidades de autoayuda, participaron el grupo "Volver a nacer" de la parroquia Nuestra Señora del Valle y "Nuestra Señora de Luján" de Florencio Varela, "La Casita" Jesús Me Guía, y el "Grupo Asunción" de Quilmes, todos integrantes de la Pastoral de Drogodependencia de la diócesis de Quilmes.

Aquí hay una mayor convergencia si pensamos en el Estado más allá de la Dirección, pues existe un sostenimiento explícito al trabajo de estos sacerdotes a partir del financiamiento estatal de muchas de las iniciativas que Ilevan adelante. Esto también se observa en la buena relación con el director provincial y con los directores municipales de culto, quienes reconocen el trabajo territorial de larga tradición en la Iglesia católica. Por su parte, la estrategia dada por esta iglesia no deja de considerar la salud y el bienestar de manera integral, incluyendo la dimensión religiosa, pero está más abierta al diálogo fluido con una concepción biomédica de salud y bienestar, reconociendo el trabajo de médicos, psicólogos y psiquiatras en la recuperación del adicto ${ }^{(46)}$.

Finalmente, también pudimos observar la presencia de instituciones que, al encontrar la explicación a la adicción en factores de índole individual y familiar, conciben el consumo como una enfermedad crónica y primaria. Estos centros, originados en la modalidad de los "doce pasos", trabajan con profesionales de la salud en actividades terapéuticas individuales y grupales. Incorporan la creencia en un Poder Superior, sin autopresentarse como "religiosos". Este tipo de tratamientos son los más reconocidos por el Estado, por medio de becas, a la vez que cuentan usualmente con cobertura por obras sociales y prepagas $^{(9)}$. La dinámica de estas organizaciones pone el énfasis en la promoción de una gestión del autocuidado de los consumidores, enlazada con formas de cuidado sociales y espirituales.

En ambas ediciones de la Expo, participó la institución Creer es Crear, así como también grupos de Alcohólicos Anónimos que se reúnen en espacios de la Iglesia católica. Consideramos que podemos incluir en este grupo al Centro Varelense de Rehabilitación Social (CEVARESO), espacio municipal de "orientación, atención y contención de adicciones". Nos interesa detenernos un momento sobre este centro, pues es el único espacio propiamente estatal a nivel local de trabajo con adicciones que encontramos en las Expo. Responde a este tercer tipo en tanto considera, en su folletería "proponer alternativas de orientación médica, psicológica y religiosa" para el adicto, como el octavo paso (de diez) para ayudar a quien consume alcohol y drogas ilegalizadas. Además, se basa en el eslogan "Fomentamos una vida saludable". El Ilamado a este tipo de vida en vínculo con los abordajes médicos, psicológicos y religiosos nos habla nuevamente del carácter multidimensional de la definición de salud, compartida, al menos en una primera instancia, tanto por actores religiosos como estatales. En este caso puntual, es el Estado el que asume la religión como parte importante de la recuperación del adicto, incorporándola como un elemento más del tratamiento. Sin embargo, podemos preguntarnos qué se entiende, en este caso, por intervenciones "religiosas", pues como hemos visto en los apartados anteriores, distintas religiones tienen diferentes concepciones sobre la salud y lo que se puede considerar una adicción, teniendo por tanto variadas formas de abordar el tratamiento.

Habiendo presentado los grupos integrantes del evento, podemos dar cuenta de una participación diversa en términos de tipo de organizaciones para el trabajo con adicciones en la Expo, reforzando la idea de la Dirección de abrir espacios de participación plural, si bien todas estas entidades pertenecen únicamente a iglesias cristianas -evangélicas y católicas-, cuyo trabajo con la atención de adicciones tiene lógicas semejantes ${ }^{(45)}$. Más allá de la pertenencia a determinada adscripción religiosa de estas comunidades, es relevante el hecho de que el Estado reconozca en ellas una expertise para gestionar el cuidado de personas con 
este tipo de problemáticas, al invitarlas a dar cuenta de las actividades que realizan, aun cuando esto no pretenda establecer el Ilamado a la formulación conjunta de políticas públicas en la materia.

En este sentido, estos espacios hacen converger concepciones del bienestar y la salud con elementos compartidos y contrapuestos al mismo tiempo. Tanto Estado como religiones ven en la adicción un problema a ser resuelto, pues atenta contra la salud y el bienestar de las personas. En ocasiones, discrepan en las formas de resolución en tanto priorizan un aspecto de la salud por sobre el otro, sea biomédico o espiritual (por mencionar solo aquellos más encontrados en nuestro trabajo de campo). En otras, se destacan los elementos compartidos al predominar concepciones "integrales" sobre las personas.

Vemos que, a la vez, no es posible hallar definiciones homogéneas y lineales sobre la salud y el bienestar ni de parte de los agentes religiosos ni de la de los estatales. De esta manera, nos interesa destacar que lo que se está poniendo en juego en estos tipos de eventos es, más allá de la diversidad religiosa existente o no, una forma de comprender la salud y el bienestar con puntos de encuentro y tensión entre actores estatales y religiosos.

\section{REFLEXIONES FINALES}

En este trabajo, nos hemos propuesto dar cuenta de las tensiones y puntos de encuentro existentes en las definiciones que agentes estatales y agentes religiosos elaboran en torno a la salud y el bienestar. Para ello introducimos el cuidado como categoría analítica en la medida en que, desde una mirada amplia e integral, nos permitió incluir la presencia de prácticas y formas de gestión en tres dimensiones distintas e interrelacionadas: una material (del cuidado corporal), una de relaciones sociales (redes de soporte y apoyo mutuo) y una espiritual (de contacto con lo numinoso y acceso al bienestar por esa vía). Más allá de las tensiones existentes, estas dimensiones del cuidado, se hallan presentes en la forma en que el Estado y las diversas religiones analizadas conciben a la salud y el bienestar. A partir de allí, observamos los eventos producidos por el Estado provincial, como forma de promoción de la salud en general y del trabajo con relación al consumo problemático de drogas ilegalizadas en particular.

Consideramos que este modo de vinculación en el espacio estudiado, implica una forma de pensar la salud por parte del Estado, dando a las religiones un espacio de participación, al resaltar las dimensiones espirituales y emocionales. Ello nos habla en cierta medida de una gestión de lo común y una política del cuidado compartida, a la vez que tensionada, por actores estatales y religiosos. En efecto, el entender que la salud de las personas no se reduce al plano físico de la noenfermedad y que, por tanto, la prevención y recuperación de personas que practican consumos problemáticos de drogas, por ejemplo, debe vincularse también con las dimensiones mencionadas es un dato no menor sobre la gestión provincial en materia de salud.

Hemos visto que, en la mayoría de los casos, existe una tendencia convergente a definir de forma multidimensional la salud y el bienestar. Sin embargo, esto no supone un acuerdo acrítico de los agentes ni la total subsunción de un actor a otro. Por eso es que, además de hablar de puntos de encuentro, sostenemos que existen tensiones en las definiciones analizadas. En la medida en que los eventos estudiados son principalmente un espacio de difusión de actividades, lo que está en cuestión no es sino una puesta discursiva sobre definiciones acerca de la salud, el bienestar y el cuidado, sin que ello implique, en la práctica, una apertura a la generación de políticas públicas que se basan en alguna de las cosmovisiones presentes en estos espacios.

Para analizar el caso de las Expo Promo Salud, hemos propuesto un análisis en tres formas particulares de intervención y gestión del cuidado por parte de instituciones religiosas que participaron del evento organizado por la Dirección Provincial de Cultos: en primer lugar, la concepción de la Iglesia 
adventista sobre el bienestar y la salud; en segundo, la definición de estos dos últimos términos por parte de la Iglesia Scientology; y en tercero, la reconstrucción de tres abordajes distintos en materia de intervención en situaciones de drogodependencia.

A partir de la literatura existente $y$, sobre todo, de lo visto en el trabajo del campo, hemos construido la noción de cuidado como una categoría conformada por tres dimensiones distintas e interrelacionadas: una material, una social/relacional y una espiritual. Veremos ahora, a modo de síntesis, cada una de estas dimensiones a la luz de los tres casos analizados en los últimos tres apartados del artículo.

En la primera edición, hemos visto a los adventistas elaborando una forma de gestión del cuidado que, iniciando con la promoción de la salud en su sentido más clásico (es decir, como salud física) promueve el ingreso a espacios colectivos que lleven finalmente al encuentro con Dios como forma de obtención de un bienestar integral. El espacio brindado por la Expo promueve la gestión de cuidado material por medio de la feria descripta en el cuerpo del texto e indirectamente colabora con el motivo evangelizador de los adventistas.

En el caso de la Iglesia Scientology, el Estado habilita un espacio de prevención y de difusión sobre las drogas en general (estén legalizadas o no). Al indagar en las formas de concebir la salud por parte de esta cosmovisión, remarcamos una tensión: a partir del cuidado, construido como esencialmente individual -cuidado de sí-, se apunta a la dimensión espiritual. La propuesta para el alcance de bienestar parte del entendimiento de que es solo mediante los principios Scientology que se llega al conocimiento del espíritu, lo que habilita prácticas de auto-cuidado y el eventual fin del consumo. Lo más interesante, sin embargo, no es tanto este "corrimiento" hacia lo espiritual sino la oposición al consumo de drogas legalizadas por el Estado. Esto para nosotras da cuenta en gran medida de las fragmentaciones presentes al interior del aparato estatal.

Finalmente, la centralidad dada por la Dirección Provincial de Cultos al tratamiento del consumo problemático de drogas supone un reconocimiento explícito de una expertise particular de las religiones (principalmente la católica y las iglesias evangélicas) sobre la temática. No obstante, esta expertise no es unívoca para las distintas instituciones que promocionan su actividad en la Expo, sino que da cuenta de distintas formas de abordaje que van desde estrategias de cuidado fuertemente ancladas en lo espiritual, pasando por dinámicas ancladas en causalidades de raigambre social, hasta finalmente, otras con causalidades y tratamientos entendidos como integrales (en aquellas comunidades terapéuticas que reciben financiamiento estatal).

Al suceder todas estas tensiones en un marco de estatalidad, hemos analizado la complejidad de las definiciones sociales sobre salud y bienestar, entendidas como categorías multidimensionales. Si por un lado hemos mostrado las variadas concepciones y elementos que hacen a los discursos de las cosmovisiones religiosas, esperamos haber dado cuenta también del carácter no homogéneo de las concepciones estatales en la materia. En efecto, las definiciones estatales sobre la salud y el bienestar, lejos de ser meramente seculares o concebidas linealmente en términos biomédicos -tal como supondría el sentido común-, nos muestran tensiones y distensiones con aquellas elaboradas por instituciones religiosas.

Así, nuestra apuesta consiste en concebir las cosmodefiniciones sobre cuidado involucradas, entendiendo que lo que está en juego no son solo cosmovisiones religiosas sino, también, cosmovisiones estatales. Esto puede permitirnos reflexionar a futuro sobre qué es lo que sucede cuando estas conceptualizaciones, aquí en el nivel discursivo, entran en pugna con la elaboración de políticas públicas. 


\section{AGRADECIMIENTOS}

Este trabajo ha sido posible gracias al financiamiento otorgado por el Consejo Nacional de Investigaciones Científicas y Técnicas (CONICET), a través de las becas doctorales iniciales de las cuales ambas autoras son beneficiarias desde 2016, bajo la dirección del doctor Alejandro Frigerio y la codirección del doctor Antonio Camou (en el caso de García Bossio) y bajo la dirección de la doctora Verónica Giménez Béliveau y la codirección de la doctora Ana Mallimaci (en el caso de Monjeau Castro). Agradecemos los comentarios de los/las evaluadores/as anónimos/as del artículo, que ayudaron profundamente a mejorar y enriquecer esta versión.

\section{REFERENCIAS BIBLIOGRÁFICAS}

1. Giménez Béliveau V. Terapéuticas católicas, males modernos: procesos de sanación y exorcismo en Argentina. Sociedad y religión. 2017;XXVII(47):33-59.

2. Viotti N, Funes ME. La política de la nueva era: el arte de vivir en Argentina. Debates do NER. 2015;2(28):17-36.

3. Viotti N, Funes ME. La búsqueda de confort en las prácticas religiosas: reseña de "Um Deus de todos os días; uma análise sobre pessoa, aflição e conforto numa trama religiosa de Buenos Aires". Sociedad y religión. 2016;26(45):184-188.

4. Irrazábal G. Religión y salud: la intervención pública de agentes religiosos católicos formados en bioética en el debate parlamentario sobre la muerte digna en la Argentina. Salud Colectiva. 2015;11(3):331-349.

5. Olmos Álvarez AL. "Venid a mí todos los afligidos": salud, enfermedad y rituales de sanación en el movimiento católico carismático del Padre Ignacio. Ciencias Sociales y Religión/Ciências Sociais e Religião. 2015;17(22):52-70.

6. Bordes M, Algranti J. El sentido de la adhesión: un análisis de la construcción de significados en el marco de situaciones sociales evangélicas y terapéuticas alternativas (Buenos Aires, Argentina). Revista Colombiana de Antropología. 2014;50(2):219-242.

7. Giumbelli E, Toniol R. What is spirituality for? New Relations between Religion, Health, and Public Spaces. En: Mapryl J, Blanes R, Giumbelli E, Wilson EK, editores. Secularisms in a postsecular age?: religiosities and subjectivities in comparative perspective. Switzerland: Palgrave Macmilla; 2017. p. $147-168$
8. Gilligan C. El daño moral y la ética del cuidado. En: Gilligan C. La Ética del Cuidado. Vol. 30. Barcelona: Cuadernos de la Fundación Víctor Grífols i Lucas; 2013. p. 12-39.

9. Camarotti AC, Güelman L, Azparren AL, Jones D, Di Leo PF. Respuestas religiosas y espirituales a los consumos de drogas en el Área Metropolitana de Buenos Aires: perspectivas y prácticas de actores institucionales. Mendoza: II Jornadas de Sociología de la Universidad Nacional de Cuyo; 2015.

10. Marcus G. Etnografía en/del sistema mundo: el surgimiento de la etnografía multilocal. Alteridades. 2001;11(22):111-127.

11. Glaser BG, Strauss A. The discovery of grounded theory: strategies for qualitative research. New York: Aldine Publishing Company; 1967.

12. Guber R. La entrevista antropológica: introducción a la no directividad. En: Guber R. El salvaje metropolitano: reconstrucción del conocimiento social en el trabajo de campo. Buenos Aires: Paidós; 2004. p. 132-142.

13. Guber R. La etnografía: Método, campo y reflexividad. Buenos Aires: Siglo Veintiuno Editores; 2012.

14. Guber R, compiladora. Prácticas etnográficas: ejercicios de reflexividad de antropólogas de campo. Buenos Aires: Instituto de Desarrollo Económico y Social, Miño y Dávila; 2014.

15. Gupta A. Fronteras borrosas: el discurso de la corrupción, la cultura de la política y el estado imaginado. En: Abrams P, Gupta A, Mitchell T. Antropología del estado. México: FCE; 2015. p. 71-144

16. Esping-Andersen G. Los tres mundos del estado de bienestar. Valencia: Institució Valenciana d'Estudis i Investigació, Ediciones Alfons El Magnànim; 1993.

17. Adelantado J, Noguera J, Rambla X, Sáez L. Las relaciones entre estructura y política sociales: una propuesta teórica. Revista Mexicana de Sociología. 1998;60(3):123-156.

18. Esquivel V, Faur E, Jelin E. Hacia una conceptualización del cuidado: familia, mercado y Estado. In: Esquivel V, Faur E, Jelin E, editores. Las lógicas del cuidado infantil: entre las familias, el Estado y el mercado. Buenos Aires: IDES; 2012.

19. Picchio A. Un enfoque macroeconómico ampliado de las condiciones de vida. Barcelona: Jornadas "Tiempos, trabajos y género"; 2001. 
20. Marco Navarro F. El cuidado de la niñez en Bolivia y Ecuador: cuidado de algunos, obligaciones de todas [Internet]. Santiago: CEPAL, Naciones Unidas; 2007 [citado 1 jul 2017]. Disponible en: https://tinyurl.com/y8n3ptay.

21. Draibe S, Riesco M. Estado de bienestar, desarrollo económico y ciudadanía: algunas lecciones de la literatura contemporánea. En: Sojo A, Uthoff A, editores. Cohesión social en América Latina y el Caribe: una revisión perentoria de algunas de sus dimensiones. Santiago: CEPAL, Naciones Unidas; 2007. p. 67-76.

22. Zibecchi C. Organizaciones comunitarias y cuidadoras: reconfiguración de responsabilidades en torno al cuidado infantil. En: Pautassi L, Zibecchi C, coordinadoras. Las fronteras del cuidado: agendas, derechos e infraestructura. Buenos Aires: Biblos; 2013. p. 317-349.

23. Santillán L. Prácticas cotidianas e intersecciones entre la Iglesia Católica y grupos familiares en asentamientos populares del Gran Buenos Aires. Cadernos de Campo. 2008;17(17):111-132.

24. Batthyány Dighiero K. Las políticas y el cuidado en América Latina: una mirada a las experiencias regionales [Internet]. Santiago: CEPAL, Naciones Unidas, Cooperación Española; 2015 [citado 1 jul 2017]. Disponible en: https://tinyurl. com/y89kqjop.

25. Tronto JC. Who cares?: how to reshape a democratic politics. Ithaca: Cornell University Press; 2015.

26. Arango LG. El trabajo de cuidado: ¿servidumbre, profesión o ingeniería emocional? En: Arango LG, Molinier P, editores. El trabajo y la ética del cuidado. Bogotá: La Carreta Editores, Universidad Nacional de Colombia; 2011. p. 91-109.

27. Paperman P. La perspectiva del care: de la ética a lo político. En: Arango LG, Molinier P, editores. El trabajo y la ética del cuidado. Bogotá: La Carreta Editores, Universidad Nacional de Colombia; 2011. p. 25-44.

28. Álvarez L, Auyero J. "La ropa en el balde": rutinas y ética popular frente a la violencia en los márgenes urbanos. Nueva Sociedad. 2014;(251):17-30.

29. Al-Mohammad H, Peluso D. Ethics and the "rough ground" of the everyday: the overlappings of life in postinvasion Iraq. HAU: Journal of Ethnographic Theory. 2012;2(2):42-58.

30. Expo Promo Salud en Solano. Hecho en Quilmes [Internet]. 4 may 2017 [citado 1 jul 2017]. Disponible en: https://tinyurl.com/ydhex8fw.
31. Dirección Provincial de Cultos. Misiones y funciones [Internet]. La Plata: Subsecretaría General de la Provincia de Buenos Aires; [citado 1 jul 2017]. Disponible en: https://tinyurl.com/ya64bvhk.

32. Mallimaci F. El mito de la Argentina laica: catolicismo, política y Estado. Buenos Aires: Capital Intelectual; 2015.

33. Frigerio A, Wynarczyk H. Diversidad no es lo mismo que pluralismo: cambios en el campo religioso argentino (1985-2000) y lucha de los evangélicos por sus derechos religiosos. Sociedade e Estado. 2008;23(2):227-260.

34. Iglesia Adventista del Séptimo Día. Entrenamiento feria de salud [Video en Internet] [citado 1 nov 2017]. Disponible en: https://tinyurl.com/ yaxa3uo6.

35. Esquivel JC. Religion and politics in Argentina: religious influence on legislative decisions on sexual and reproductive rights. Latin American Perspectives. 2016;43(3):133-143.

36. Ministerio de la Salud de la Iglesia Adventista del Séptimo Día. Manual de la Feria de Salud [Internet]. Buenos Aires: Ministerio de la Salud de la Iglesia Adventista del Séptimo Día; 2014 [citado 30 nov 2017]. Disponible en: https://tinyurl.com/ y9w7ec6j.

37. Iglesia Scientology Argentina. ¿Qué es scientology? [Internet]. Buenos Aires: Iglesia Scientology Argentina; [citado 30 nov 2017]. Disponible en: https://tinyurl.com/y8ts2t8r.

38. Garbi S. La administración de la palabra en las comunidades terapéuticas. En: Epele M, compiladora. Padecer, cuidar y tratar: estudios socioantropológicos sobre consumo problemático de drogas. Buenos Aires: Editorial Antropofagia; 2013. p. 153-184.

39. Garbi SL, Touris MC, Epele M. Técnicas terapéuticas y subjetivación en tratamientos con usuarios/as de drogas. Ciência \& Saúde Coletiva 2012;17(7):1865-1874.

40. Castilla MV, Lorenzo G. Consumo de pasta base/paco, prácticas de rescate y religiosidad pentecostal. Sociedad y religión. 2013;23(39):54-78.

41. Sánchez Antelo V. La dimensión temporal del consumo de drogas: análisis sociológico desde una categoría clave para el estudio de los procesos de salud-enfermedad-atención-cuidado. Salud Colectiva. 2016;12(1):41-54.

42. Mosqueira M. Religión y toxicomanías juveniles: sentidos y prácticas terapéuticas de agentes 
evangélicos orientados al tratamiento de jóvenes en consumos problemáticos de drogas en Argentina. Buenos Aires: IX Jornadas Internacionales Ciencias Sociales y Religión "La institución como proceso: configuraciones de lo religioso en las sociedades contemporáneas"; 2017.

43. Mosqueira M, Algranti J, Rocca L. "Institucionalidades transversales": albores y devenires institucionales de ministerios evangélicos orientados al tratamiento de usuarios de drogas en la Argentina. Buenos Aires: IX Jornadas Internacionales Ciencias Sociales y Religión "La institución como proceso: configuraciones de lo religioso en las sociedades contemporáneas"; 2017.
44. Carbonelli M. Pan y palabras: la inserción evangélica en la gestión pública en Argentina. Religião e Sociedade. 2015;35(2):73-95.

45. Jones D, Cunial S. Más allá de los límites del Estado: instituciones católicas y evangélicas de partidos del Gran Buenos Aires (Argentina) en la implementación de políticas públicas sobre drogas. Desafíos. 2017;29(2):85-123.

46. Lucesole MJ, Marina R. Curas villeros: en la cruzada por recuperar a los adictos. La Nación [Internet]. 12 may 2015 [citado 5 dic 2017]. Disponible en: https://tinyurl.com/ycqsnetv.

\section{FORMA DE CITAR}

García Bossio MP, Monjeau Castro C. Entre las religiones y el Estado: el caso de las Expo Promo Salud en la provincia de Buenos Aires. Salud Colectiva. 2018;14(2):323-340. doi: 10.18294/sc.2018.1531.

Recibido: 16 de julio de 2017 | Versión final: 21 de diciembre de 2017 | Aprobado: 26 de diciembre de 2017

Este obra está bajo una licencia de Creative Commons Reconocimiento-NoComercial 4.0

Internacional. Reconocimiento - Permite copiar, distribuir y comunicar públicamente la obra.

A cambio, se debe reconocer y citar al autor original. No Comercial - Esta obra no puede ser

utilizada con finalidades comerciales, a menos que se obtenga el permiso.

http://dx.doi.org/10.18294/sc.2018.1531 